Debabrata Ghosh*

\title{
IMPACT OF THE COVID-19 PANDEMIC ON THE EXPRESSION OF EMOTIONS IN SOCIAL MEDIA
}

DOI: $10.22367 / \mathrm{mcdm} .2020 .15 .02$

Received: 7.01.2021 | Revised: 25.04.2021 | Accepted: 14.09.2021.

\begin{abstract}
In the age of social media, every second thousands of messages are exchanged. Analyzing those unstructured data to find out specific emotions is a challenging task. Analysis of emotions involves evaluation and classification of text into emotion classes such as Happy, Sad, Anger, Disgust, Fear, Surprise, as defined by emotion dimensional models which are described in the theory of psychology (www 1; Russell, 2005). The main goal of this paper is to cover the COVID-19 pandemic situation in India and its impact on human emotions. As people very often express their state of the mind through social media, analyzing and tracking their emotions can be very effective for government and local authorities to take required measures. We have analyzed different machine learning classification models, such as Naïve Bayes, Support Vector Machine, Random Forest Classifier, Decision Tree and Logistic Regression with 10-fold cross validation to find out top ML models for emotion classification. After tuning the Hyperparameter, we got Logistic regression as the best suited model with accuracy $77 \%$ with the given datasets. We worked on algorithm based supervised ML technique to get the expected result. Although multiple studies were conducted earlier along the same lines, none of them performed comparative study among different ML techniques or hyperparameter tuning to optimize the results. Besides, this study has been done on the dataset of the most recent COVID-19 pandemic situation, which is itself unique. We captured Twitter data for a duration of 45 days with hashtag \#COVID19India OR \#COVID19 and analyzed the data using Logistic Regression to find out how the emotion changed over time based on certain social factors.
\end{abstract}

Keywords: classification, COVID-19, emotion, emotion analysis, Naïve Bayes, Pandemic, Random Forest, SVM.

\footnotetext{
Tata Consultancy Services, Kolkata, India, e-mail: debabrata.g@tcs.com, debag.edu@gmail.com.
} 


\section{Introduction}

Emotion analysis is an advanced and fine grained version of sentiment analysis. Many surveys and papers have been published on sentiment analysis. Sentiment analysis provides the information about the positive, negative or neutral categories, but it requires more detailed and granular level analysis of the information. For example: 'It was a bad luck! We lost the game' vs. 'we lost the game because of the worst decision of the umpire'; here both sentences are of negative sentiment, but the first sentence is of 'sadness', whereas the second sentence is of 'anger'.

Emotion analysis fills this gap with more detailed analysis of human emotion (www 1; Russell, 2005). Emotion can be expressed through different medium, such as textual message, voice or facial expressions; this is a huge area of research work. There are multiple studies going on in each of these specific areas to decipher the human emotions and translate them to actionable forms.

Emotion analysis data can be used in multiple industry segments. Products reviews and customer feedbacks can be analyzed in different emotion categories, which helps the merchant to improve the product line or propose a suitable product to the customer to improve customer satisfaction. Social media messages can be analyzed to understand the mood and needs of the people of a particular locality or demography, which helps companies to target promotions. In the healthcare sector, emotion analysis can be helpful to diagnose the mental health of a patient through analysis of social media blogs, tweets, messages; it can also predict the sudden mood swings of people that result in suicidal attempts. In the public sector, emotion analysis can be helpful in election campaign, etc. Our study addresses the fluctuation of human emotions in the COVID-19 pandemic situation across India.

Analysis of Twitter data or the thoughts of the crowd over a long period of time presents a very clear picture and provides insightful information about the mentality of the common people. This helps the government or management to take appropriate measures in time. The COVID-19 pandemic has affected the socio-economic condition of the common people very badly and it has had a deep effect on the mental situation of the common people. This study can be performed in a specific state or area; people very often share their thoughts in social media; analyzing this textual information can be very helpful to predict forthcoming situations, so that administration can take proper measures to tackle unpredicted situations. 
The rest of the paper is organized as follows: in Section 2, we discuss related work. In Section 3, we present detailed steps of our proposed approach. In Section 4, we present the analysis of the Twitter data and a visualization of the results. In Section 5, we present conclusions and recommendations.

\section{Objective of the study and the novelty of the work}

The main goal of this paper is to cover the COVID-19 pandemic situation in India and its impact on human emotions. As people very often express their state of the mind through social media, analyzing and tracking their emotions can be very effective for the government and local authorities to take required measures. We have analyzed different machine learning classification models, such as Naïve Bayes, Support Vector Machine, Random Forest Classifier, Decision Tree and Logistic Regression with 10-fold cross validation to find out the top ML models for emotion classification. After tuning the Hyperparameter, we got Logistic regression as the best suited model with accuracy $77 \%$ with the given datasets. We used algorithms based supervised ML technique to get the expected result. Although multiple studies were conducted earlier in the same line, none of them performed a comparative study among different ML techniques or hyperparameter tuning to optimize the results. Besides, this study has been done on the dataset of the most recent COVID-19 pandemic situation, which is itself unique.

\section{Literature review}

With ever increasing popularity of different social media and microblogging sites, it is of paramount interest for different researchers to analyze and construct meaningful insights from the messages. Wiebe, Wilson and Cardie (2005) worked on manual annotation of expressions of opinions and emotions in language for the corpus size of 10,000. Arora et al. (2010) worked on the automatic feature construction using subgraph mining algorithm. Gupta, Gilbert and Di Fabbrizio (2012) worked on emotion detection in email customer care using salient features and N-gram. Mohammad and Turney (2010) worked on the creation of an emotion lexicon using Amazon's Mechanical Turk. Keshtkar and Inkpen (2010) worked on a technique to extract paraphrases for emotion terms from non-parallel corpora. Lee, Chen and Huang (2010) worked on emotion cause detection using a text-driven rule based system. Ramanand, Bhavsar and Pedanekar (2010) worked on finding actionable insights from suggestions and 'buy' wishes of product reviewers using a lexicon-based 
approach. Volkova et al. (2010) worked on an emotion analysis system, showing how an annotation task can be set up. Hanser et al. (2010) worked on enhancing news reading experiences by integrating 30 seconds long Flash animations into news article web pages depicting their content and emotional aspects. Bandhakavi et al. (2017) worked on general-purpose emotion lexicons (GPELs) that associate words with emotion categories. Gupta et al. (2020) introduced a compact and efficient way of representing text for further downstream natural language processing (NLP) tasks. Kumar, Kawahara and Kurohashi (2018) proposed a two-layered attention network based on Bidirectional Long ShortTerm Memory for sentiment analysis. Hakak et al. (2017) summarized the previous studies of textual emotion analysis based on various emotional models and computational approaches used. Yanran et al. (2017) developed a highquality multi-turn dialog dataset. Similar work was done by Hakak et al. (2019). Ahmed et al. (2020) proposed an attention-based model to detect emotion from tweets. Gupta et al. (2017) worked on a sentiment and semantics based approach for emotion detection in textual conversations.

\section{Methodology}

\subsection{Proposed approach of emotion classification process}

As shown in Figure 1, we have taken JIRA and Stack Overflow labeled dataset for training and testing purposes. We applied different preprocessing techniques to remove noise and unwanted words or characters. Then we did feature extraction using TF-IDF and N-Gram techniques. In the next step, we used five different classification algorithm with 10 -fold cross validation to find out the top three algorithms, then tuned the hyperparameters to get the best algorithm for the given dataset.

In parallel to the above mentioned process, we were collecting Twitter data related to \#COVID19 for 45 days. After preprocessing, we fed the Twitter data in the selected machine learning model to classify the tweets into five different emotion categories. 


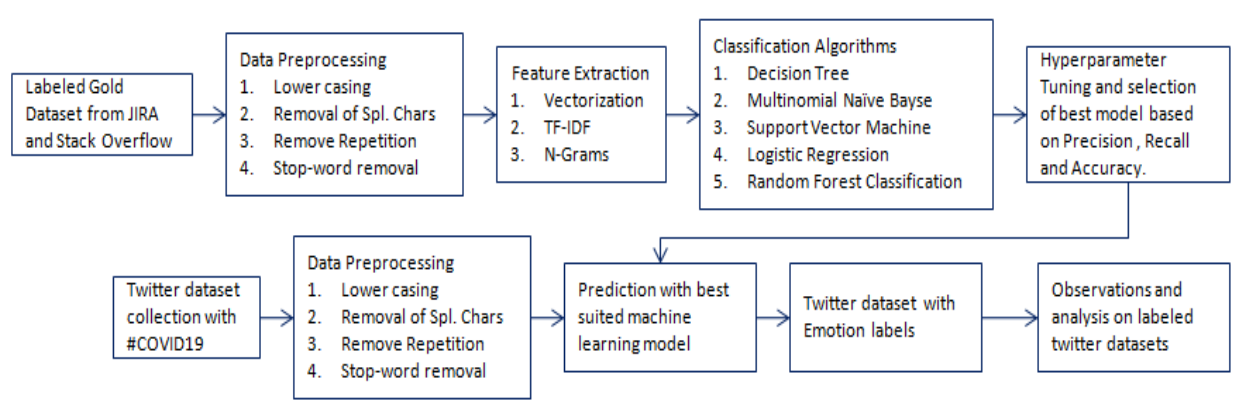

Figure 1: Proposed approach of emotion classification process

\subsection{Emotion selection for pandemic situation}

Emotion classification requires choosing the most appropriate model to categorize human sentiments. Researchers mostly classify emotions on the basis of the framework proposed by Shaver et al. (1987) or on Ekman's six basic emotions (Ekman, 1971). In this study, we used two gold standards datasets annotated according to the discrete framework by Shaver et al. (1987). This framework defines a tree-structured hierarchical classification of emotions, where each level refines the granularity of the previous one, thus providing more indication about its nature. It includes six basic top-level emotions, namely: love, joy, anger, sadness, fear, and surprise. The gold standard datasets had very small leveled data for fear and surprise categories; in the COVID-19 situation the fear factor is very closely related to sadness, and surprise is quite similar to joy or happiness. So here we are broadly categorizing the emotions in four main categories: love, joy, anger, and sadness.

\subsection{Training and testing dataset}

Multiple research papers in related fields were consulted to get the understanding and to obtain the proven Gold standard dataset to model the system, but, unfortunately, there are very limited data sets available for the emotion types labeled. Besides, most of the datasets are crowd source data, so the degree of correctness varies depending on the person and the situations. Also on the basis of multiple feedbacks from different researchers, we have selected the labeled dataset of stack overflow and JIRA as the gold standard dataset. These datasets have been annotated according to the discrete framework by Shaver et al. (1987). It includes six basic emotions, namely: love, joy, anger, sadness, fear, and surprise. But the 'surprise' category was not considered very often as labeled datasets. Table 1 shows the different emotion categories of 
labeled data collected from Stack Overflow and JIRA. Figure 2 shows the distribution of the dataset under different emotion categories. It is observed that the majority of the datasets fall under the category of 'Love' and 'Anger'.

Table 1: Gold standard labeled Training Dataset

\begin{tabular}{|l|r|r|r|r|r|}
\hline \multirow{2}{*}{ Dataset } & \multicolumn{6}{|c|}{ Emotion Level Distribution of the Gold Dataset } \\
\cline { 2 - 6 } & Love & \multicolumn{1}{c|}{ Joy } & \multicolumn{1}{c|}{ Anger } & \multicolumn{1}{c|}{ Sadness } & \multicolumn{1}{c|}{ Fear } \\
\hline Stack OF & 1220 & 491 & 882 & 230 & 106 \\
\hline JIRA & 166 & 124 & 324 & 302 & 0 \\
\hline Total & 1386 & 615 & 1206 & 532 & 106 \\
\hline
\end{tabular}
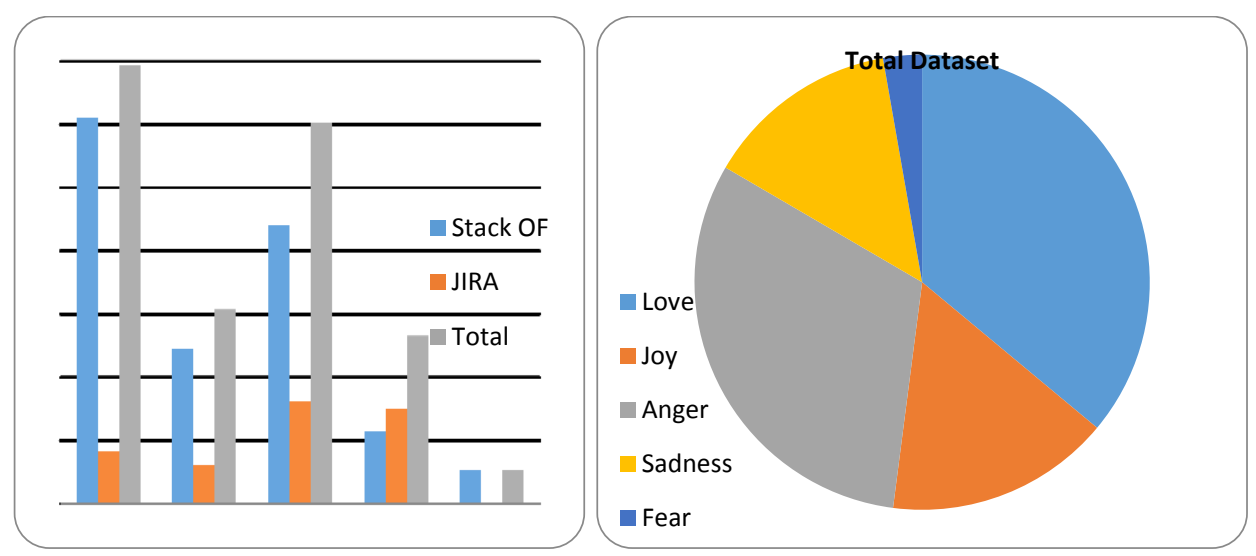

Figure 2: Distribution of the dataset under different emotion category

The distribution of the emotion categories is not uniform. So there might be biasness towards the majority of the dataset. There are five categories of emotions: Love, Joy, Anger, Sadness and Fear.

\subsection{Social Media Data Collection for emotion analysis}

We collected Twitter (www 2) data for a duration of 45 days starting from $15^{\text {th }}$ Oct, 2020 to $30^{\text {th }}$ Nov, 2020. We used tweepy API with geolocation as the central location of India and radius of $1000 \mathrm{~km}$ to collect Twitter data with the hashtag \#COVID19India OR \#COVID19. As India is one of the countries most affected by COVID-19, we collected data from all over India to get a sense how emotions are changing over a period of 45 days. We collected 2000 tweets every day and ignored all re-tweets; in total, we collected a corpus of 80000 tweets for emotion classification. We only considered tweets written in English and we used tweet_mode as extended to collect tweets with a limit of 280 characters. 


\subsection{Data preprocessing}

Figure 3 shows the steps for data preprocessing.

- Lowercasing: Entire text has been converted to lower case letter.

- Remove special chars: Special characters such as @,',http ,^ etc. have been replaced by spaces.

- Remove repetitions: Repetition of the words have been removed.

- Transform short negation form: Short negation forms, such as can't, have been replaced by cannot.

- Remove stop words: Stop words have been removed using Python NLTK library.

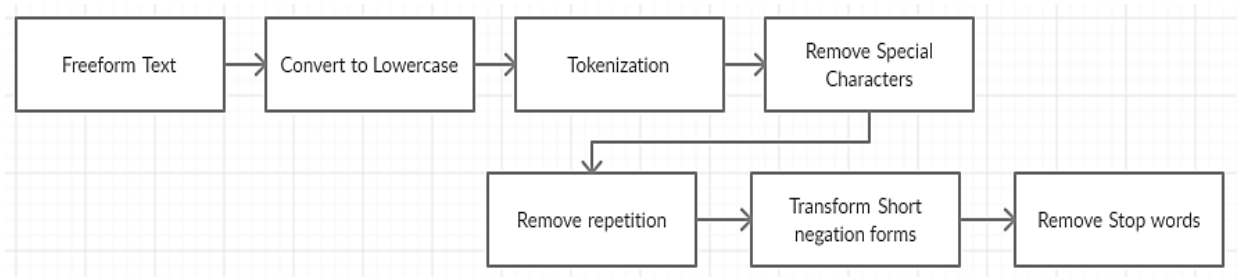

Figure 3: Flow diagram - data preprocessing steps

\subsection{Feature extraction}

We removed all the 'English' stop words and used wordnet lemmatization before proceeding with tokenization. We used CountVectorizer for vector representation of the sentences with word frequency as integer number. It built a vocabulary with the top features ordered by term frequency across the corpus. We used TF-IDF and n-gram for feature extraction. For n-gram, we tried unigram, bi-gram, and tri-gram to get the best accuracy. It was found that the combination of unigram and bi-gram provided the best result.

\subsection{Classification techniques and comparative study}

We did a comparative study of the five different machine learning classification techniques with default parameter setting and with 10 -fold cross validations. As shown in Table 2, the results of different ML techniques were compared on the basis of precision, recall, F1-score and accuracy. The experimental results captured in Table 2 show that the top three ML models with high accuracy are SVM, LR, and RFC. These three ML models have been tuned to get better results; details of the hyperparameter tuning are given in the next section. 
Table 2: Comparative study of different machine learning models

\begin{tabular}{|l|l|l|l|l|l|l|l|l|l|l|l|l|l|l|l|l|}
\hline \multirow{2}{*}{ Emotions } & \multicolumn{3}{|c|}{ Decision Tree } & \multicolumn{4}{c|}{ MNB } & \multicolumn{4}{c|}{ SVM } & \multicolumn{2}{c|}{ Logistic Regression } & \multicolumn{3}{c|}{ RFC } \\
\cline { 2 - 16 } & P & R & F1 & P & R & F1 & P & R & F1 & P & R & F1 & P & $R$ & F1 \\
\hline anger & 0.8061 & 0.7336 & 0.7681 & 0.7381 & 0.9066 & 0.8137 & 0.6957 & 0.9412 & 0.8001 & 0.7065 & 0.9412 & 0.8071 & 0.7905 & 0.8616 & 0.8245 \\
\hline fear & 0.3667 & 0.3793 & 0.3729 & 0 & 0 & 0 & 0.5001 & 0.0691 & 0.1212 & 0 & 0 & 0 & 0.4615 & 0.2069 & 0.2857 \\
\hline joy & 0.3191 & 0.4111 & 0.3593 & 0.5 & 0.0274 & 0.0519 & 0.5001 & 0.1849 & 0.2701 & 0.6545 & 0.2466 & 0.3582 & 0.405 & 0.3356 & 0.3571 \\
\hline love & 0.7421 & 0.7191 & 0.7304 & 0.6435 & 0.9635 & 0.7717 & 0.7221 & 0.8315 & 0.7728 & 0.7273 & 0.8539 & 0.7855 & 0.7321 & 0.8062 & 0.7674 \\
\hline sadness & 0.7647 & 0.7324 & 0.7482 & 0.9545 & 0.4437 & 0.6058 & 0.9223 & 0.6691 & 0.7755 & 0.9029 & 0.6549 & 0.7592 & 0.8264 & 0.7042 & 0.7605 \\
\hline Accuracy & & & 0.6684 & & & 0.6985 & & & 0.7193 & & & 0.7328 & & & 0.7183 \\
\hline micro avg & 0.5997 & 0.5951 & 0.5958 & 0.5672 & 0.4682 & 0.4486 & 0.6681 & 0.5391 & 0.5479 & 0.5982 & 0.5393 & 0.5421 & 0.6431 & 0.5829 & 0.6011 \\
\hline wt. avg & 0.6891 & 0.6684 & 0.6773 & 0.6766 & 0.6985 & 0.6273 & 0.7033 & 0.7193 & 0.6854 & 0.7141 & 0.7328 & 0.6996 & 0.7058 & 0.7183 & 0.7082 \\
\hline
\end{tabular}

\subsection{Hyperparameter tuning}

Each of the identified models can be tuned with different parameters as follows:

Logistic Regression: This ML model has been tuned with the parameters (www 3) from Table 3.

Table 3: Logistic Regression Hyperparameters setup

\begin{tabular}{|l|l|l|}
\hline \multicolumn{3}{|c|}{ Logistic Regression Hyperparameters setup } \\
\hline \multicolumn{1}{|c|}{ Hyperparameters } & \multicolumn{1}{c|}{ Test Value sets } & \multicolumn{1}{c|}{ Selected value set } \\
\hline C & $1,2.78,7.74$ & 2.78 \\
\hline max_iter & $1000,10000,50000$ & 50000 \\
\hline penalty & 11,12 & 11 \\
\hline norm & 11,12 & 12 \\
\hline use_idf & True, False & TRUE \\
\hline ngram_range & $(1,1),(1,2),(2,2)$ & $(1,2)$ \\
\hline
\end{tabular}

Support Vector Machine: This ML model has been tuned with the parameters (www 4) from Table 4.

Table 4: SVM Hyperparameters setup

\begin{tabular}{|l|l|l|}
\hline \multicolumn{2}{|c|}{ SVM Hyperparameters setup } \\
\hline \multicolumn{1}{|c|}{ Hyperparameters } & \multicolumn{1}{c|}{ Test value sets } & \multicolumn{1}{c|}{ Selected value set } \\
\hline C & $1,10,100,1000$ & 1 \\
\hline degree & $2,3,4,5$ & 2 \\
\hline gamma & $1,0.1,0.01,0.001,0.0001$ & 1 \\
\hline kernel & rbf','linear' & linear \\
\hline norm & 11,12 & 12 \\
\hline use_idf & True, False & FALSE \\
\hline ngram_range & $(1,1),(1,2),(2,2)$ & $(1,2)$ \\
\hline
\end{tabular}


RFC: This ML model has been tuned with the parameters (www 5) from Table 5 .

Table 5: RFC Hyperparameters setup

\begin{tabular}{|l|l|l|}
\hline \multicolumn{2}{|c|}{ RFC Hyperparameters setup } \\
\hline \multicolumn{1}{|c|}{ Typerparameters } & \multicolumn{1}{c|}{ Test Value sets } & \multicolumn{1}{c|}{ Selected value set } \\
\hline max_depth & $10,25,50,100$ & 25 \\
\hline min_samples_leaf & 2,5 & 2 \\
\hline min_samples_split & $2,3,5,10$ & 5 \\
\hline n_estimators & $10,100,200,500$ & 500 \\
\hline norm & 11,12 & 12 \\
\hline use_idf & True, False & FALSE \\
\hline ngram_range & $(1,1),(1,2),(2,2)$ & $(1,1)$ \\
\hline
\end{tabular}

\subsection{The best selected ML model}

Table 6 shows the comparative study of the three ML models after hyperparameters tuning was performed for each of them. The comparison was based on precision, recall, F1-score and accuracy. It was found that the logistic regression has the highest accuracy score of $77 \%$; the accuracy score has been improved by $3.6 \%$ after parameter tuning.

Table 6: Comparative study of three ML models after hyperparameter tuning

\begin{tabular}{|l|c|r|c|c|c|c|c|c|c|}
\hline \multirow{2}{*}{ Emotions } & \multicolumn{3}{|c|}{ RFC } & \multicolumn{3}{c|}{ SVM } & \multicolumn{3}{c|}{ Logistic Regression } \\
\cline { 2 - 11 } & P & \multicolumn{1}{c|}{ R } & F1 & P & R & F1 & P & R & F1 \\
\hline anger & 0.7071 & 0.9273 & 0.8024 & 0.7479 & 0.9239 & 0.8266 & 0.7946 & 0.9101 & 0.8484 \\
\hline fear & 0 & 0 & 0 & 0.5001 & 0.0691 & 0.1212 & 0.6154 & 0.2759 & 0.3811 \\
\hline joy & 0.7143 & 0.1712 & 0.2762 & 0.6338 & 0.3082 & 0.4147 & 0.6588 & 0.3836 & 0.4848 \\
\hline love & 0.7035 & 0.9129 & 0.7946 & 0.7238 & 0.8539 & 0.7835 & 0.7476 & 0.8736 & 0.8057 \\
\hline sadness & 0.9651 & 0.5845 & 0.7281 & 0.9001 & 0.6972 & 0.7857 & 0.8718 & 0.7183 & 0.7876 \\
\hline Accuracy & & & $\mathbf{0 . 7 2 8 7}$ & & & $\mathbf{0 . 7 4 5 3}$ & & & $\mathbf{0 . 7 6 9 2}$ \\
\hline macro avg & 0.6181 & 0.5192 & 0.5203 & 0.7011 & 0.5704 & 0.5864 & 0.7376 & 0.6323 & 0.6615 \\
\hline wt. avg & 0.7236 & 0.7287 & 0.6845 & 0.7366 & 0.7453 & 0.7209 & 0.7626 & 0.7692 & 0.7544 \\
\hline
\end{tabular}

\section{Classification of Twitter data and visualization of results}

\subsection{Testing results and analysis of the classified emotions with dates}

We collected Twitter data for a duration of 45 days with \#COVID19India OR \#COVID19, collecting a set of 2000 tweets per day. In the current pandemic situation, with the unavailability of proper medications so far and the number of 
cases not yet under control, people are mostly angry and frustrated. In addition, job loss due to a long lockdown period, and the overall social economic condition in India is very bad. Still, news of the vaccines against COVID-19, gradual removal of lockdown, and economic recovery raised some hope and created a positive mindset among the people. This complete picture has been reflected in our analysis of the data captured in a span of more than one month. Close to $80 \%$ of the tweets showing anger and frustration related to the COVID-19 situation, there are certain small ups and downs in the graph but over all people are angry towards the COVID-19 condition.

\subsection{Visualization of results}

In Figure 4, we show a comparison of different emotions: anger, fear, joy, love and sadness, over a period of 45 days and their fluctuations with time and different social-economic factors.

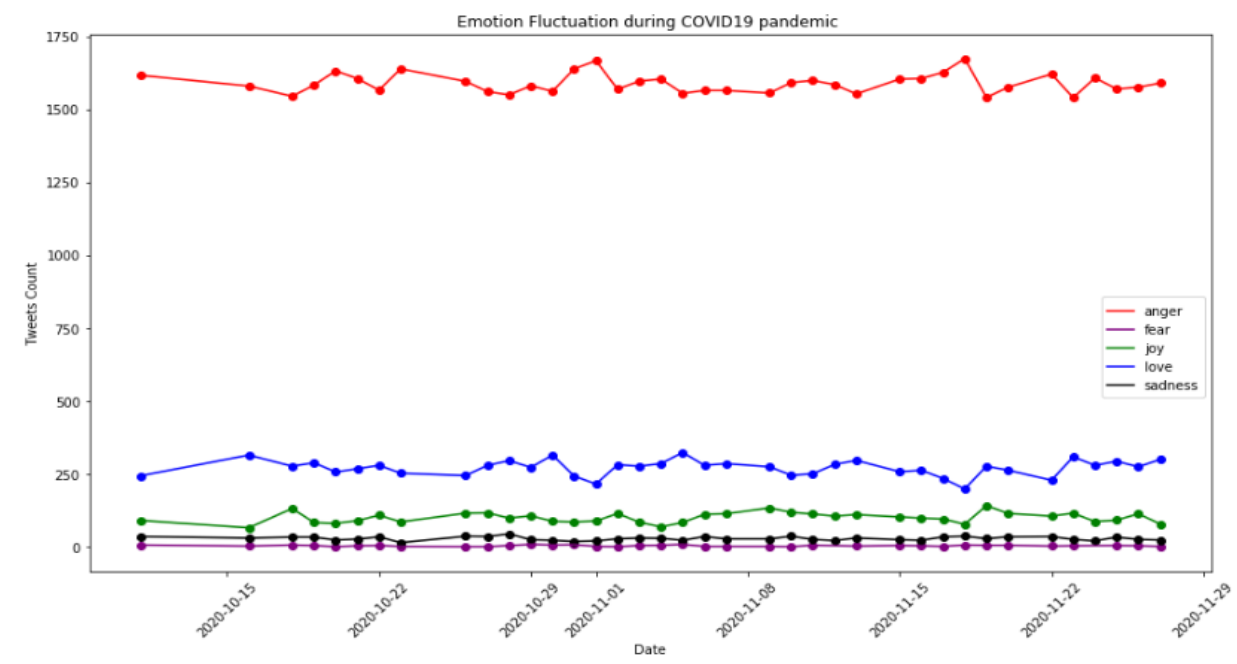

Figure 4: Emotion fluctuation during the COVID-19 pandemic

From the above plot it is evident that the 'anger' factor has dominated other emotions. In Figure 5, we have plotted the 'anger' emotion separately to get more detailed information about its trends. It can be seen that the anger factor is gradually decreasing of late. 


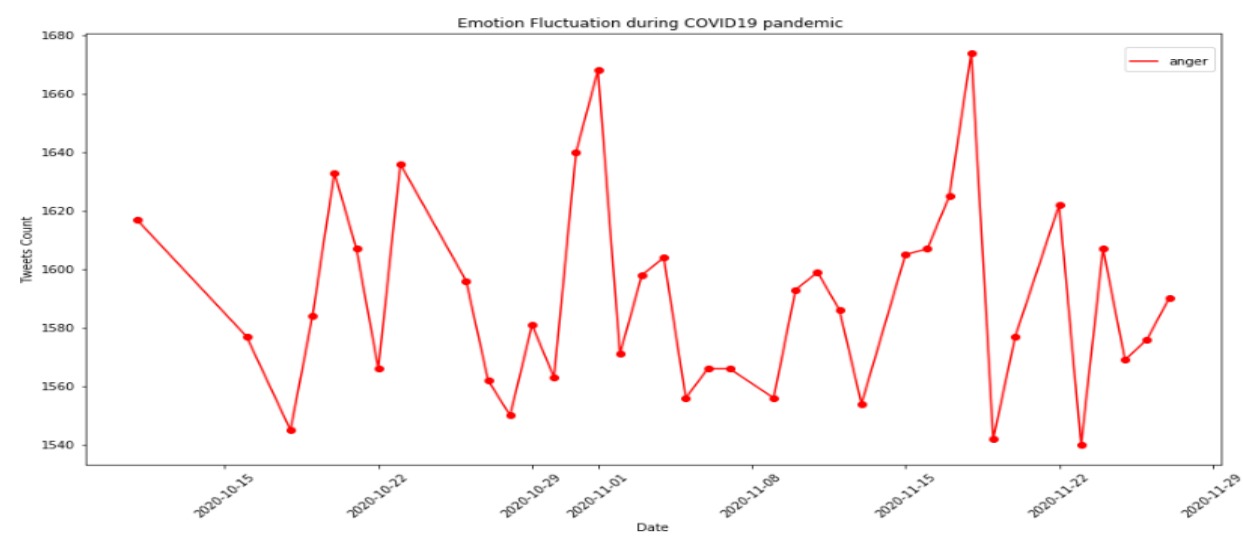

Figure 5: Variation of 'Anger' during the COVID-19 pandemic

\section{Conclusion and recommendations}

In this paper, we have done a comparative study of Decision Tree (DT), Multinomial Naive Bayes (MNB), Support Vector Machine (SVM), Random Forest Classifier (RFC) and Logistic Regression (LR) machine learning classification techniques. On the basis of precision, recall, F-score and accuracy, we selected the SVM, RFC and LR techniques for further performance optimization. Then we tuned the required parameter of the SVM, RFC and LR techniques and found that LR is the best technique with an accuracy of $77 \%$ for the given datasets. We collected tweets related to COVID-19 across India for a period of 45 days, and using the machine learning technique identified we classified the tweets into five different emotion categories. It has been observed that the 'anger' factor dominated the other emotions in COVID-19 related tweets. The selection technique of the ML model and its implementation can be used in a small local area to deeply analyze the mood swings of people in that area. Also, we discussed the application of this technique in other industry segments or for socio-political purposes. In future, this system can be extended to automatically identify other emotion categories in a more granular way and apart from depending on the small set of labeled dataset from crowd sourcing. This research study can be extended to decipher not only the contextual meaning of the messages, but also to perform psychological analysis of the users.

\section{Declaration}

1. Funding: Not Applicable

2. Conflicts of interest/Competing interests: Not Applicable

3. Availability of data and material: Available

4. Code availability: Available 


\section{References}

Ahmed S., Reyadh A., Sithil F., Shah F., Shaafi A. (2020), An Attention-based Approach to Detect Emotion from Tweets, 182-187, DOI: 10.1109/IC2IE50715.2020.9274600.

Arora S., Mayfield E., Penstein-Rosé C., Nyberg E. (2010), Sentiment Classification Using Automatically Extracted Subgraph Features, Proceedings of the NAACL HLT 2010 Workshop on Computational Approaches to Analysis and Generation of Emotion in Text, Association for Computational Linguistics, Los Angeles, 131-139.

Bandhakavi A., Wiratunga N., Massie S., Deepak P. (2017), Lexicon Generation for Emotion Detection from Text, IEEE Intelligent Systems, 32, 102-108, DOI: 10.1109/MIS.2017.22.

Chang Y.-C., Yeh W.-C., Hsing Y.-C., Wang C.-A. (2019), Refined Distributed Emotion Vector Representation for Social Media Sentiment Analysis, PloS ONE, 14(10), 1-22.

Ekman P. (1971), Universals and Cultural Differences in Facial Expressions of Emotion, Nebraska Symposium on Motivation, 19.

Gupta N., Gilbert M., Di Fabbrizio G. (2012), Emotion Detection in Email Customer Care, Computational Intelligence, 29, 10-16, DOI: 10.1111/j.1467-8640.2012.00454.x.

Gupta S., Kanchinadam T., Conathan D., Fung G. (2020), Task-Optimized Word Embeddings for Text Classification Representations, Frontiers in Applied Mathematics and Statistics, 5, 67, DOI: 10.3389/fams.2019.00067.

Gupta U., Chatterjee A., Srikanth R., Agrawal P. (2017), A Sentiment-and-Semantics-Based Approach for Emotion Detection in Textual Conversations, https://arxiv.org/abs/1707.06996.

Hakak N., Kirmani M., Mohd M., Ahmed M., Mohsin M. (2019), Automatic Emotion Classifier: Proceedings of ICACIE 2017, Volume 1, Progress in Advanced Computing and Intelligent Engineering, 565-572, DOI: 10.1007/978-981-13-1708-8_52.

Hakak N., Mohd M., Kirmani M., Mohd M. (2017), Emotion Analysis: A Survey, 2017 International Conference on Computer, Communications and Electronics (Comptelix), 397-402, DOI: 10.1109/COMPTELIX.2017.8004002.

Hanser E., McKevitt P., Lunney T., Condell J. (2010), NewsViz: Emotional Visualization of News Stories, Proceedings of the NAACL HLT 2010 Workshop on Computational Approaches to Analysis and Generation of Emotion in Text, 125-130.

Keshtkar F., Inkpen D. (2010), A Corpus-based Method for Extracting Paraphrases of Emotion Terms, Workshop on Computational Approaches to Analysis and Generation of Emotion in Text, 35-44.

Kumar A., Kawahara D., Kurohashi S. (2018), Knowledge-enriched Two-layered Attention Network for Sentiment Analysis, Proceedings of the 2018 Conference of the North American Chapter of the Association for Computational Linguistics: Human Language Technologies, Vol. 2 (Short Papers), 253-258.

Lee S., Chen Y., Huang C.-R. (2010), A Text-driven Rule-based System for Emotion Cause Detection, Proceedings of the NAACL HLT 2010 Workshop on Computational Approaches to Analysis and Generation of Emotion in Text, 45-53.

Li Y., Su H., Shen X., Li W., Cao Z., Niu S. (2017), DailyDialog: A Manually Labelled Multi-turn Dialogue Dataset, https://arxiv.org.pdf/1710.03957.pdf.

Mohammad S., Turney P. (2010), Emotions Evoked by Common Words and Phrases: Using Mechanical Turk to Create an Emotion Lexicon, Proceedings of the NAACL HLT 2010 Workshop on Computational Approaches to Analysis and Generation of Emotion in Text.

Ovesdotter Alm C., Roth D., Sproat R. (2005), Emotions from Text: Machine Learning for Textbased Emotion Prediction, Proceedings of the Conference on Human Language Technology and Empirical Methods in Natural Language Processing, 579-586. 
Pearl L., Steyvers M. (2010), Identifying Emotions, Intentions, and Attitudes in Text Using a Game with a Purpose, Proceedings of the NAACL HLT 2010 Workshop on Computational Approaches to Analysis and Generation of Emotion in Text, Association for Computational Linguistics, 71-79.

Potamias R., Siolas G., Stafylopatis A. (2020), A Transformer-based Approach to Irony and Sarcasm Detection, Neural Computing and Applications, 32, DOI: 10.1007/s00521-02005102-3.

Ramanand J., Bhavsar K., Pedanekar N. (2010), Wishful Thinking: Finding Suggestions and 'Buy' Wishes from Product Reviews, Proceedings of the NAACL HLT 2010 Workshop on Active Learning for Natural Language Processing, 54-61.

Russell J.A. (2005), Emotion in Human Consciousness Is Built in Core Affect, Journal of Consciousness Studies, 12, 8-10, 26-42.

Shaver P., Schwartz J., Kirson D., O’Connor C. (1987), Emotion Knowledge: Further Exploration of a Prototype Approach, Journal of Personality and Social Psychology, 52(6), 1061-1086.

Strapparava C., Valitutti A. (2004), WordNet-Affect: An Affective Extension of WordNet, Proceedings of the Fourth International Conference on Language Resources and Evaluation (LREC'04), 1083-1086.

Volkova E., Mohler B., Meurers D., Gerdemann D., Bülthoff H., Inkpen D., Strapparava C. (2010), Emotional Perception of Fairy Tales: Achieving Agreement in Emotion Annotation of Text, Proceedings of the NAACL HLT 2010 Workshop on Computational Approaches to Analysis and Generation of Emotion in Text, 98-106.

Wiebe J., Wilson T., Cardie C. (2005), Annotating Expressions of Opinions and Emotions in Language, Language Resources and Evaluation, 39(2-3), 165-210.

(www 1) Types Emotions and Their Effect on Human Behavior, https://www.verywellmind. com/an-overview-of-the-types-of-emotions-4163976 (accessed: 5.04.2021).

(www 2) Information collected from social networking site, www.twitter.com (accessed: 5.10.2020).

(www 3) Qiao F., Logistic Regression Model Tuning with Scikit-learn, https://towardsdata science.com/(accessed: 8.01.2019).

(www 4) Dawson C., SVM Parameter Tuning, https://towardsdatascience.com/ (accessed: 31.05.2020).

(www 5) Koehrsen W., Hyperparameter Tuning the Random Forest in Python, https://towards datascience.com/ (accessed: 10.01.2018). 